Manuscript prepared for the

Proceedings of the $24^{\text {th }}$ International Symposium on Discharges and Electrical Insulation in Vacuum

(ISDEIV)

Braunschweig, Germany, August 30-Sept 3, 2010

\title{
A Seemingly Simple Task: Filling a Solenoid Volume in Vacuum with Dense Plasma
}

\author{
André Anders ${ }^{1}$, Marina Kauffeldt ${ }^{2}$, Prabir Roy ${ }^{1}$, and Efim Oks ${ }^{3}$ \\ ${ }^{1}$ Lawrence Berkeley National Laboratory, 1 Cyclotron Road, Berkeley, California 94720, USA \\ ${ }^{2}$ University of the German Federal Armed Forces, Werner-Heisenberg-Weg 39, 85577 Neubiberg, Germany \\ ${ }^{3}$ High Current Electronics Institute, 2/3 Academichesky Ave, Tomsk, 634055, Russia
}

Manuscript completed June 24, 2010.

\section{DISCLAIMER}

This document was prepared as an account of work sponsored in part by the United States Government. While this document is believed to contain correct information, neither the United States Government nor any agency thereof, nor The Regents of the University of California, nor any of their employees, makes any warranty, express or implied, or assumes any legal responsibility for the accuracy, completeness, or usefulness of any information, apparatus, product, or process disclosed, or represents that its use would not infringe privately owned rights. Reference herein to any specific commercial product, process, or service by its trade name, trademark, manufacturer, or otherwise, does not necessarily constitute or imply its endorsement, recommendation, or favoring by the United States Government or any agency thereof, or The Regents of the University of California. The views and opinions of authors expressed herein do not necessarily state or reflect those of the United States Government or any agency thereof or The Regents of the University of California. 


\title{
A Seemingly Simple Task: Filling a Solenoid Volume in Vacuum with Dense Plasma
}

\author{
André Anders ${ }^{1}$, Marina Kauffeldt ${ }^{2}$, Prabir Roy ${ }^{1}$, and Efim Oks ${ }^{3}$ \\ ${ }^{1}$ Lawrence Berkeley National Laboratory, 1 Cyclotron Road, Berkeley, California 94720, USA \\ ${ }^{2}$ University of the German Federal Armed Forces, Werner-Heisenberg-Weg 39, 85577 Neubiberg, Germany \\ ${ }^{3}$ High Current Electronics Institute, 2/3 Academichesky Ave, Tomsk, 634055, Russia
}

\begin{abstract}
Space-charge neutralization of a pulsed, high-current ion beam is required to compress and focus the beam on a target for warm dense matter physics or heavy ion fusion experiments. We described attempts to produce dense plasma in and near the final focusing solenoid through which the ion beam travels, thereby providing an opportunity for the beam to acquire the necessary charge-compensating electrons. Among the options are plasma injection from four pulsed vacuum arc sources located outside the solenoid, and using a high current $(>4 \mathrm{kA})$ pulsed vacuum arc plasma from a ring cathode near the edge of the solenoid. The plasma distribution is characterized by photographic means and by an array of movable Langmuir probes. The plasma is produced at several cathode spots distributed azimuthally on the ring cathode. Beam neutralization and compression are accomplished, though issues of density, uniformity, and pulse-to-pulse reproducibly remain to be solved.
\end{abstract}

\section{INTRODUCTION}

For warm dense matter and ion-beam-driven inertial fusion experiments, high current pulsed ion beams can be used to heat and compress a target of interest. While many beams will be needed for inertial fusion, current experiments aim at the development of a single beam of heavy ions such as $\mathrm{K}^{+}$or $\mathrm{Li}^{+}$(here, "heavy" is understood in comparison to the mass of protons). The ion beam needs to be compressed radially and longitudinally to reach on target a current in the $\mathrm{kA}$ range and with a pulse duration of $\sim 1 \mathrm{~ns}[1]$. A very strong space charge would build up unless a means of ion space charge compensation is provided.

Space charge compensation can be achieved when the ion beam travels through plasma whose density exceeds the beam density. Therefore, linear heavy ion accelerators designs incorporate a plasma generator placed near and/or inside the finial focusing solenoid (FFS). In previous efforts, this included a ferroelectric plasma source upstream of the FFS, and four cathodic arc plasma sources injecting plasma from downstream of the FFS [2-3]. Results obtained at the Neutralized Drift Compression Experiment (NDCX) showed 60-fold longitudinal compression of a $300 \mathrm{keV} \mathrm{K}^{+}$beam [2-3].

The next generation accelerator experiment, NDCX-II [4], currently under construction at Berkeley

Work at Berkeley Lab was supported by the U.S. Department of
Lab, requires improved neutralization for higher beam currents.

In this contribution we will start with a brief summary of the previous effort involving four pulsed vacuum arc sources. Then we will introduce an approach involving a ring cathode vacuum arc source placed inside the FFS. The resulting plasma distribution will be characterized by photographic and probe techniques.

\section{SOLENOID FILLING WITH PLASMA FROM A SET OF}

\section{FOUR VACUUM ARC PLASMA SOURCES}

The idea of filling the solenoid is based on using streaming vacuum plasma, produced outside the solenoid, and injecting it along the magnetic field lines into the solenoid (Fig. 1). For symmetry reasons, four miniature plasma sources are used, which had previously been developed for pulsed cathodic arc deposition of thin films [5]. Fig. 2 shows the sources on a common mounting flange, and Fig. 3 shows the plasma in on-axis view.

It is clear that a number of issues have to be resolved towards the goals of filling the volume of the solenoid with dense plasma. Among them are the magnetic mirror effect [6] and the limited availability of magnetic field lines that do not intersect the vacuum wall and other components. The latter point is clearly illustrated in Fig 1: following the field lines from the source one can see that only the central region of the solenoid is filled with field lines not intersecting the wall, and hence only the central axial region should receive dense plasma. This has been investigated using a movable array of 37 Langmuir probes (Fig. 4) [7]. Each of the probes was individually biased to record the time-dependent ion saturation current. It was found that the plasma inside the solenoid is concentrated near the axis of symmetry, with a density maximum of up to $4 \times 10^{19} \mathrm{~m}^{-3}$ for an arc current of 770 A per source. The maximum field strength in the center of the solenoid was $8 \mathrm{~T}$. The plasma "filament" inside the solenoid had a cross section much smaller than the cross section of the ion beam to be neutralized. Hence there is a need for a different approach. Variation of the plasma source positions would not help. The seemingly simple task turns out to be quite difficult in the presence of a strong field! 


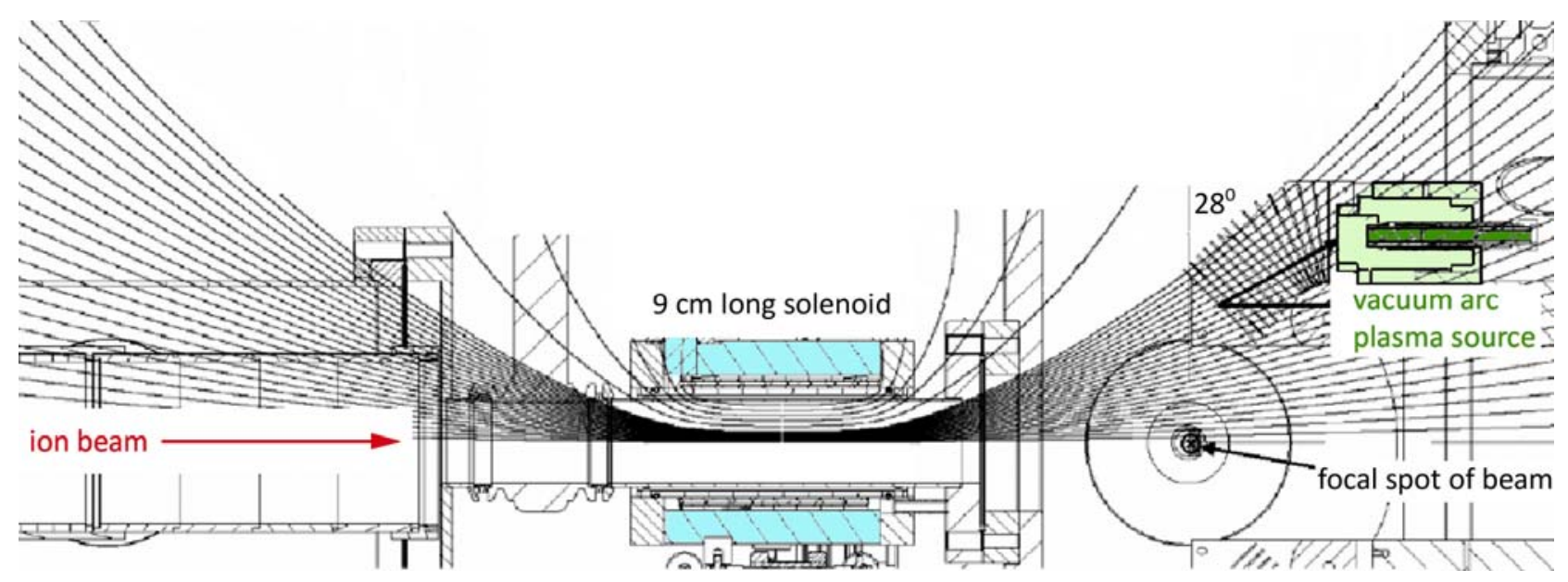

Fig. 1 Magnetic field lines of the final focusing solenoid, with the ion beam coming from the left and one of the plasma sources indicated on the right.

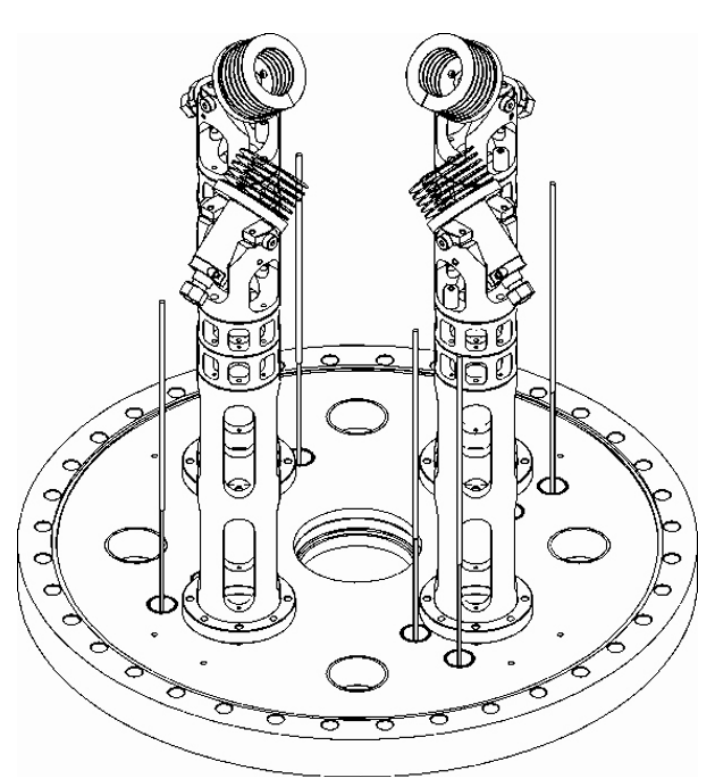

Fig. 2 Set of four plasma sources, each tilted $28^{\circ}$ to the axis as to inject plasma along magnetic field lines.

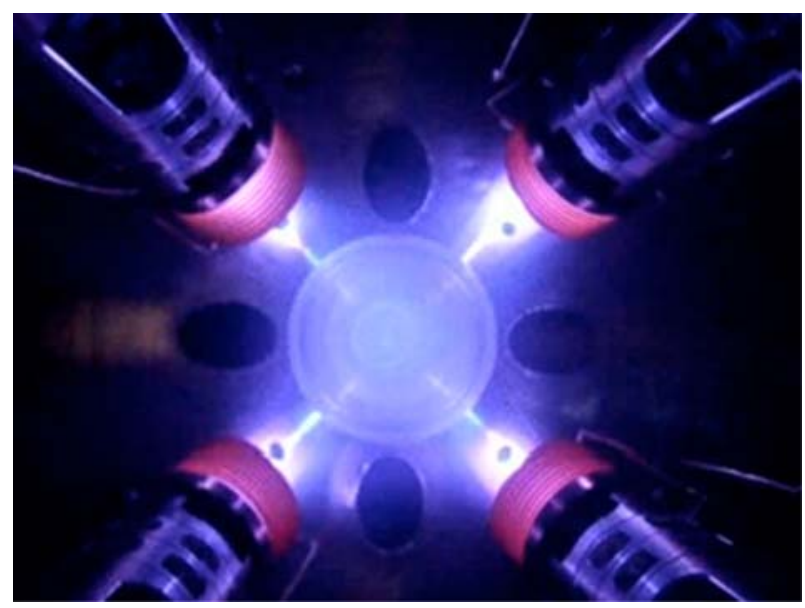

Fig. 3 On-axis view through a vacuum window, against the beam direction, with the four vacuum arc plasma sources delivering metal plasma.

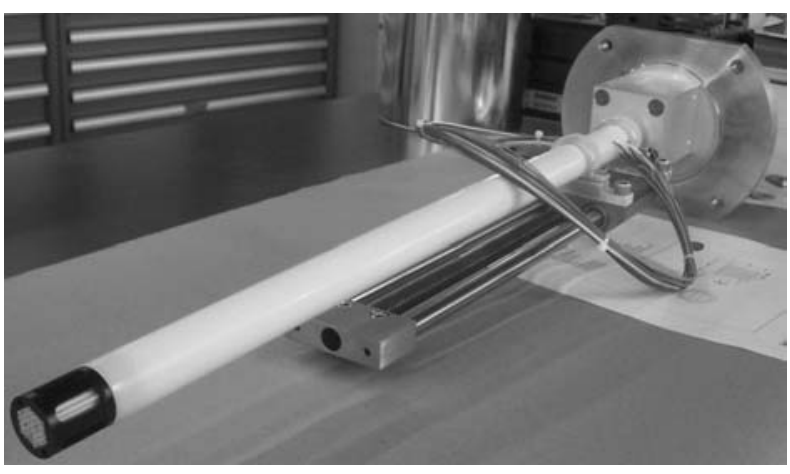

Fig. 4 Array of 37 probes mounted on an axially movable shaft.

An attempt is made to fundamentally change the plasma distribution inside the FFS by generating dense plasma inside the FFS, as opposed to injecting it from the outside. To clearly see the differences between the approaches, the same array of 37 probes was used for characterization of the new approach. We will show that the plasma generated inside the FFS has a tendency to be hollow, representing a compliment to the previously obtained plasma "filament". This suggests using a combination of hollow plasma and injected plasma to obtain a practical solution for optimum space charge compensation.

\section{SOLENOID FILLING WITH PLASMA FROM A RING-CATHODE VACUUM ARC SOURCE}

Vacuum arc plasma is an attractive option because cathode spots are known to be a prolific generator of dense metal plasma that can pump itself by condensing on the chamber walls [8-9].

In the present experiments, arc plasma was generated on a ring cathode inside the vacuum pipe which in turn was mounted inside the solenoid (Figs. 1 and 5). 


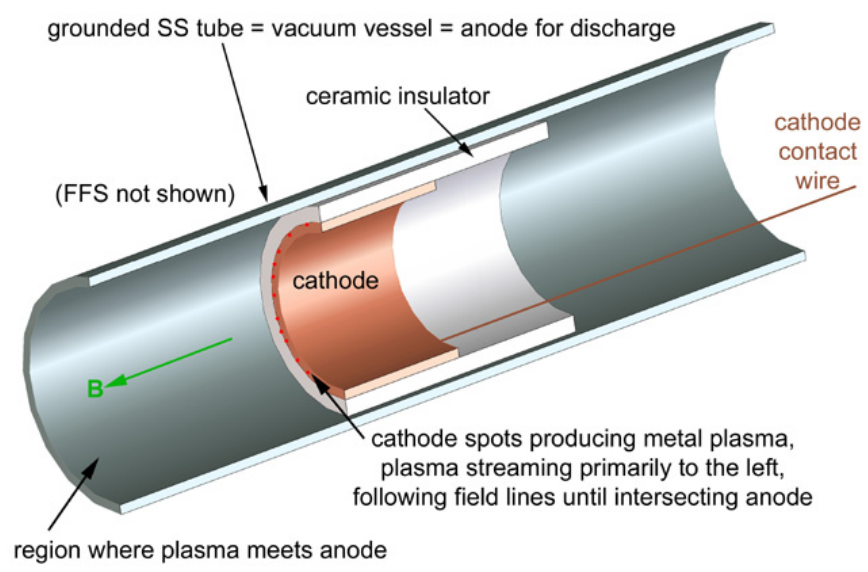

Fig. 5 Cross section of the ring-cathode cathodic arc plasma source placed inside the final focusing solenoid (not shown here but in Fig. 1).

The grounded vacuum pipe served as the anode of the discharge, and the cathode ring was separated from the pipe by a ceramic (alumina) tube. The inner diameter of the cathode ring was $25 \mathrm{~mm}$, which is large enough to accommodate the ion beam as it enters the FFS region. Initial arc ignition is facilitated by a slightly conductive layer on the front face of the insulator separating cathode and anode [10]. The plasma density is highly non-uniform: it is extremely dense near the spot, $\sim 10^{20} \mathrm{~cm}^{-3}$, and expands with $\sim 10^{4} \mathrm{~m} / \mathrm{s}$ into the vacuum.

The magnetic field for these experiments was generated by a pulsed high current of up to $8.4 \mathrm{kA}$, delivering a magnetic induction in the center of the coil up to 2.9 Tesla. For NDCX-II, this field is planned to be increased to 8 Tesla. The arc current pulse was about $200 \mu$ s and thus much shorter than the solenoid pulse, a half-sine wave of $1 \mathrm{~ms}$ FWHM. The arc current was fed by a thyristor-switched pulse-forming network. The flat portion of the arc pulse had a maximum of $4.5 \mathrm{kA}$ when the PFN was charged to the maximum of $1 \mathrm{kV}$. The arc discharge was delayed relative to the solenoid pulse such as to operate at maximum magnetic field. During the arc pulse, the magnetic field can be considered to be approximately constant. We note that the ion beam (not actually used in these experiments) has a duration of several nanoseconds, and therefore both arc plasma and magnetic field can be considered constant on that timescale. The pulse repetition rate was less than 1 pulse per second, mostly limited by the recharging time of the capacitors and cooling concerns of the FFS coil. The cathode material could be selected freely and may represent one component that can be optimized in later studies. For the preliminary studies we used aluminum in the four miniature sources and copper and stainless steel for the ring cathode experiments.

The cathode ring was slit in one place along the axial direction in order to prevent an azimuthal current that would be induced when pulsing the coil. Without the slit the cathode ring would represent a single-turn secondary of a transformer where the solenoid coil is the primary. The current in the ring is highly undesirable because its magnetic field would weaken the beam-focusing field.

\section{Plasma Diagnostics For Ring Cathode}

\section{ARRANGEMENT}

\section{A. Photography}

Photography gives semi-quantitative and intuitively clear information on the plasma distribution. The images were taken with a digital Canon EOD 300D single lens reflex camera equipped with a $200 \mathrm{~mm}$ zoom lens. The camera was operated in open shutter (bulb) mode to capture light integrated over one full discharge pulse. At relatively low arc current, less than $1 \mathrm{kA}$, only a few spots appeared and the plasma was not azimuthally uniform. Higher current, such as $4.5 \mathrm{kA}$, produced more spots around the ring periphery and hence the plasma was azimuthally distributed. When the magnetic field was applied, the plasma followed the field lines and "arched" towards the anode. The resulting image obtained by end-on photography, Fig. 6, shows a hollow plasma that fills the near-wall region.

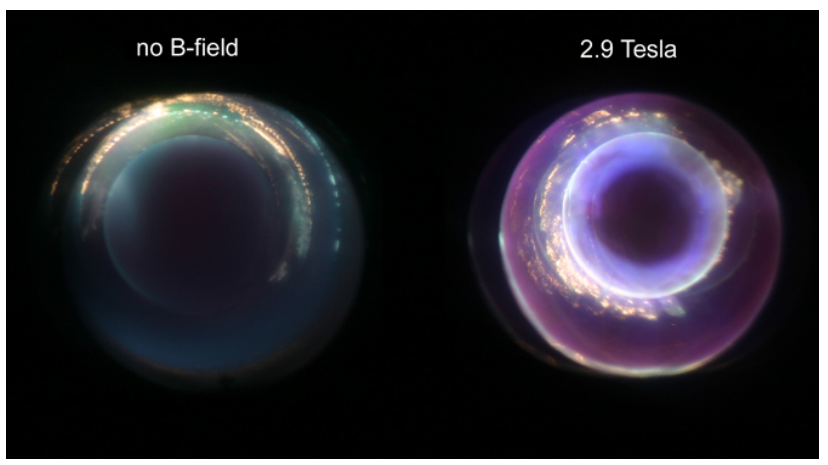

Fig. 6 Open shutter photograph of the copper plasma produced with an arc current of $4.5 \mathrm{kA}$; left: in the absence of a magnetic field, and right: at a magnetic induction of 2.9 Tesla.

\section{B. Measurements with the movable probe array}

The "hollow" plasma was also studied with the array of 37 Langmuir probes already shown in Fig. 4 and described in ref. [7]. Briefly, thirty-seven 24-gauge solid copper wires were placed in alumina tubes of 1.64 $\mathrm{mm}$ inner diameter and $2.46 \mathrm{~mm}$ outer diameter; they were arranged in a honeycomb pattern with a spacing between the centers of the wires of $2.46 \mathrm{~mm}$. The assembly could be moved axially via a precision translational stage. Each probe was connected to its circuit via a vacuum feedthrough.

The data recording was done with a National Instruments PXI-1045 chassis equipped with five PXI-5105 8-channel digitizer cards, with a maximum 
sampling rate of $60 \mathrm{MS} / \mathrm{second}$ per channel, operated under NI LabVIEW virtual instrument (VI) software. The VI allowed us to set record length, sampling rate, voltage range, and specify the trigger mode. The on-board stored data were transferred to a personal computer for further analysis using a MATLAB script.

The software was written to create equi-density contour plots and animated sequences of the plasma density evolution in one plane, or the time-averaged plasma density in selected planes of the $(\mathrm{x}, \mathrm{y}, \mathrm{z})$ space, where $\mathrm{z}$ is assigned to the axis and $(\mathrm{x}, \mathrm{y})$ are planes perpendicular to $\mathrm{z}$. When time-averaging was applied for a given fixed axial $(\mathrm{z})$ position of the probes, the averaging was done over the period between the half rise and half fall value of the cathodic arc current pulse.

Since the flowing plasma is closely bound to the magnetic field lines, it is important to place the source of plasma, the cathode spots, at a suitable location such as to guide the plasma to regions where needed. In general, the further the ring of cathode spots is placed away from the center of the solenoid the closer the plasma can arch toward the center axis. Figure 7 shows one example of measurements with the cathode edge $6.5 \mathrm{~cm}$ from the solenoid center, and the probe array positioned $3.5 \mathrm{~cm}$ from the center in the opposing direction (i.e. plasma is streaming towards the probe array). One can clearly see the hollow structure of the plasma distribution.

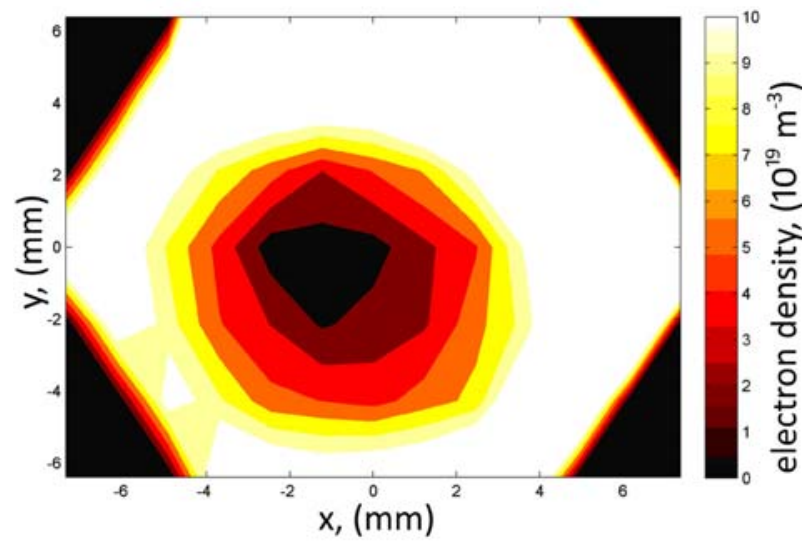

Fig. 7 Electron density distribution in the $(\mathrm{x}, \mathrm{y})$ plane at $z=3.5 \mathrm{~cm}$ measured by the 37 probe array at time $t=118 \mu$ s after discharge start; the white color indicates a density of $10^{20} \mathrm{~m}^{-3}$ or greater; arc current $4.5 \mathrm{kA}$, magnetic induction $2.9 \mathrm{~T}$.

The "hole" seen in figure 7 can be greatly reduced by further removing the ring cathode away from the center of the solenoid. The peak electron density readily exceeds the desired $10^{19} \mathrm{~m}^{-3}$, which is greater than the ion beam density. In fact, the peak electron density that has been reached was between 2 and $4 \times 10^{20} \mathrm{~m}^{-3}$, far exceeding any other previous approach to filling the solenoid volume with plasma. However, the previous approach with the four external plasma sources (Figs. 2 and 3) suggests using a combination of the two, namely filling the axis region with plasma from the sources outside the solenoid, while filling the near-wall region with plasma from the ring electrode positioned at an optimized position, i.e. some distance away from the center of the solenoid. The approximate optimum position appears to be about $8 \mathrm{~cm}$ from the center for our configuration. The remaining non-uniformity of the filling may be acceptable because we can guarantee that when the nanosecond ion beam passes the solenoid, the electron density is greater than the ion beam density at all positions inside the solenoid, and thus space charge compensation can occur. With this argument, we can also accept that the arc plasma is generally "noisy", i.e. fluctuates in time and space due to the explosive, fractal nature of plasma production at cathode spots [9].

\section{ACKNOLEDGMENTS}

Support by E. Gilson, P. Seidl, W. Greenway, M. Dickinson, S. Lidia, J.-Y. Jung, G. Logan, J. Wallig, and J. Schein are gratefully acknowledged. M.K. thanks for financial support by the Deutscher Akademischer Austauschdienst (DAAD), E.M.O. was supported by project T2-196 of the Initiatives for Proliferation Prevention of the U.S. Department of Energy.

\section{REFERENCES}

[1] P. A. Seidl, A. Anders, F. M. Bieniosek, et al. "Progress in beam focusing and compression for warm-dense matter experiments," Nucl. Instrum. Meth. Phys. Res. A, vol. 606, pp. 75-82, 2009.

[2] P. K. Roy, S. S. Yu, E. Henestroza, et al., "Drift compression of an intense neutralized ion beam," Phys. Rev. Lett., vol. 95, pp. 234801-4, 2005.

[3] A. B. Sefkow, R. C. Davidson, E. P. Gilson, et al., "Simulations and experiments of intense ion beam current density compression in space and time," Phys. Plasmas, vol. 16, pp. 056701-11, 2009.

[4] P. A. Seidl, J. Armijo, D. Baca, et al., "Plans for longitudinal and transverse neutralized beam compression experiments, and initial results from solenoid transport experiments," Nucl. Instrum. Meth. Phys. Res. A, vol. 577, pp. 215-222, 2007.

[5] R. A. MacGill, M. R. Dickinson, A. Anders, O. R. Monteiro, and I. G. Brown, "Streaming metal plasma generation by vacuum arc plasma guns," Rev. Sci. Instrum., vol. 69, pp. 801-803, 1998.

[6] F. F. Chen, Plasma Physics and Controlled Fusion. New York: Plenum Press, 1984.

[7] P. K. Roy, P. A. Seidl, et al. "A space-charge-neutralizing plasma for beam drift compression," Nucl. Instrum. Meth. Phys. Res. A, vol. 606, pp. 22-30, 2009.

[8] R. L. Boxman, D. M. Sanders, and P. J. Martin, Eds., Handbook of Vacuum Arc Science and Technology. Park Ridge, N.J.: Noyes Publications, 1995.

[9] A. Anders, Cathodic Arcs: From Fractal Spots to Energetic Condensation. New York: Springer, 2008.

[10] A. Anders, I. G. Brown, R. A. MacGill, and M. R. Dickinson, "'Triggerless' triggering of vacuum arcs," J. Phys. D: Appl. Phys., vol. 31, pp. 584-587, 1998.

E-mail of corresponding author: aanders@lbl.gov 